\section{Questions of criteria: Reply to Peterzell (1997)}

\author{
STEPHEN D. CHRISTMAN \\ University of Toledo, Toledo, Ohio \\ FREDERICK L. KITTERLE \\ Northerm Illinois University, De Kalb, Illinois \\ and \\ CHRISTOPHER L. NIEBAUER \\ University of Toledo, Toledo, Ohio
}

In a reply to our report on hemispheric differences in processing band-pass filtered letters (Christman, Kitterle, \& Niebauer, 1997), Peterzell (1997) argues that our results are not attributable to hemispheric asymmetries in spatial frequency processing. Rather, Peterzell argues that factors such as response criteria and stimulus visibility can account for our results. We argue that our results are attributable (at least in part) to hemispheric asymmetries in spatial frequency processing, while at the same time we acknowledge the potential influence of other factors in the determination of hemispheric differences.

In a reply to our report on hemispheric asymmetries in the identification of band-pass filtered letters (Christman, Kitterle, \& Niebauer, 1997), Peterzell (1997) argues that the visual field $\times$ spatial frequency interactions reported by Christman et al. are not attributable to hemispheric asymmetries in spatial frequency processing. This argument is based on two primary criteria: (1) Peterzell's characterization of the interactions involving visual field and spatial frequency as weak, unsystematic, and confusing in nature, and (2) the role of response biases, and hemispheric differences therein, in producing our effects. While we acknowledge the validity of Peterzell's points, we argue that they are insufficient for the wholesale rejection of the spatial frequency hypothesis as Peterzell advocates.

With regard to Peterzell's (1997) characterization of our results as "small, unsystematic, confusing interactions" (p. 286), we offer two counterarguments. First, we agree with Peterzell that the magnitude of our obtained effects involving visual field are relatively small, especially in comparison with the robust visual field $\times$ spatial frequency interactions that we have previously found in our lab using sinusoidal stimuli (e.g., Christman, Kitterle, \& Hellige, 1991; Kitterle, Christman, \& Conesa, 1993; Kitterle, Christman, \& Hellige, 1990; Kitterle, Hellige, \& Christman, 1992). However, the spatial frequency hypothesis, as first proposed by Sergent (1982) and later elaborated by others (e.g., Kitterle \& Christ-

Correspondence should be address to: S. D. Christman, Department of Psychology, University of Toledo, Toledo, $\mathrm{OH} 43606$ (e-mail: schrist2 @uoft02.utoledo.edu). man, 1991), simply states that the hemispheres differ in processing high versus low spatial frequencies; it does not claim that spatial frequency content is the sole determinant of hemispheric asymmetry, nor does it specify the exact magnitude of such effects. Given the accepted notion that there is no single, unifying dichotomy underlying all hemispheric asymmetries, we should not be surprised that experiments in which many factors operate yield more complicated patterns of results than do experiments in which very few factors operate. For instance, in the aforementioned work employing sinusoidal gratings, the only factor varying across stimuli was spatial frequency; all other factors such as contrast, orientation, luminance, and so forth were held constant. In this situation, spatial frequency content seems to be a strong determinant of hemispheric asymmetry. However, the use of band-pass filtered letters (as in Christman et al., 1997) introduces a whole host of other factors (e.g., orientation and phase differences among frequency components, local luminance cues, explicit pattern recognition requirements, etc.) that might each independently influence hemispheric processing. In this situation, it is not surprising that the influence of spatial frequency content is attenuated, because it is now just one voice in the crowd.

Peterzell (1997) describes our results as "unsystematic," suggesting that they might best be considered Type I errors; indeed, he points out (as did we in our original paper) that, had a more conservative criterion of $\alpha=.01$ been employed, only one of our interactions involving visual field and spatial frequency would have been deemed significant. However, an alternative interpretation is simply that, following the line of argument in the preceding paragraph, the marginal significance of our effects reflects a diminution of effect size, and not evidence for a true lack of effects of spatial frequency (cf. Rosenthal \& Rubin, 1985). Similarly, if Peterzell's (1997) contention that our visual field $\times$ spatial frequency interactions were merely Type I errors is true, one is left with the puzzling issue of why, out of eight such interaction effects with $p$ values of .10 or less, six were in the direction predicted by the spatial frequency hypothesis, two were ambiguous (i.e., Experiment 1, small letters, RT and $d^{\prime}$ data: no visual field differences at 4.0 or $16.0 \mathrm{cpd}$ and LVF advantages at $8.0 \mathrm{cpd}$ ), and none were opposite to our predictions. However, we agree that even the supportive interactions seem rather capricious, being limited, for example, to just targets and/or certain letter sizes (e.g., Experiment 1, RT to large letters).

We now come to the issue of what is determining the hemispheric asymmetries that Peterzell (1997) claims we and other researchers have been mistakenly attributing to spatial frequency. Our response is to point out that the spatial frequency hypothesis is not mutually exclusive with other accounts that Peterzell suggests. First and foremost, we are in complete agreement that response criteria play an important role and need to be taken into 
account. However, given our use of signal detection analyses, it is not clear how our results (especially our $d^{\prime}$ results) can be attributed to response bias, since the whole raison d'etre of signal detection analyses is to separate out sensitivity $\left(d^{\prime}\right)$ from response criteria $(\beta)$. Indeed, Peterzell himself equivocates on this point, stating at one point that it is "equally likely" that our results reflect true perceptual differences versus differences in response criteria, and then going on a few sentences later to state that response biases may "provide the sole explanation" of our results (p. 287). Finally, Peterzell (1997) suggests that the "visibility" hypothesis (e.g., Michimata \& Hellige, 1987), which argues that any decrease in stimulus visibility will result in preferential impairment of left hemisphere processing, provides an adequate account of our results. However, numerous reports of decreases in visibility (e.g., decreases in size, high-pass filtering) resulting in greater impairment of right hemisphere processing (e.g., Christman, 1987; Christman et al., 1997; Michimata \& Hellige, 1987) pose serious difficulties for the visibility hypothesis, but are readily accounted for by the spatial frequency hypothesis.

In conclusion, we agree with Peterzell (1997) that there is more to the determination of hemispheric asymmetries in perceptual processing than spatial frequency content alone, with factors such as stimulus perceptibility and response biases having important potential influences on hemispheric processing. However, we disagree with Peterzell's conclusion that there is no evidence for hemispheric asymmetries in spatial frequency processing; the preceding arguments notwithstanding, we would like to see how Peterzell would account for our results with sinusoidal stimuli (e.g., Christman et al., 1991; Kitterle et al., 1993; Kitterle et al., 1990; Kitterle et al., 1992) in terms of any factor other than spatial frequency. The last sentence of Peterzell's (1997) abstract states: "The results [of Christman et al., 1997] are not attributable to hemispheric asymmetries in spatial fre- quency processing"; we suggest that this be amended with the addition of a single word: "The results are not solely attributable to hemispheric asymmetries in spatial frequency processing."

\section{REFERENCES}

Christman, S. D. (1987). Effects of perceptual quality on hemispheric asymmetries in visible persistence. Perception \& Psychophysics, 41, 367-374.

Christman, S. [D.], Kitterle, F. L., \& Hellige, J. [B]. (1991). Hemispheric asymmetry in the processing of absolute versus relative spatial frequency. Brain \& Cognition, 16, 62-73.

Christman, S. D., Kitterle, F. L., \& Niebauer, C. L. (1997). Hemispheric asymmetries in the identification of band-pass filtered letters. Psychonomic Bulletin \& Review, 4, 277-284.

Kitterle, F. L., \& Christman, S. [D.] (1991). Hemispheric symmetries and asymmetries in the processing of sine-wave gratings. In F. L. Kitterle (Ed.), Advances in cerebral laterality research: Data and theory (pp. 201-224). Hillsdale, NJ: Erlbaum.

Kitterle, F. L., Christman, S. [D.], \& Conesa, J. (1993). Hemispheric differences in the interference among components of compound gratings. Perception \& Psychophysics, 54, 785-793

Kitterle, F. L., Christman, S. [D.], \& Hellige, J. B. (1990). Hemispheric differences are found in the identification, but not the detection, of low versus high spatial frequencies. Perception \& Psychophysics, 48, 297-306.

Kitterle, F. L., Hellige, J. B., \& Christman, S. [D.] (1992). Hemispheric asymmetries depend on which spatial frequencies are task relevant. Brain \& Cognition, 20, 308-314.

Michimata, C., \& Hellige, J. B. (1987). Effects of blurring and stimulus size on the lateralized processing of nonverbal stimuli. Neuropsychologia, 25, 397-407.

Peterzell, D. H. (1997). Hemispheric symmetries in the identification of band-pass filtered letters: Reply to Christman et al. (1997). Psychonomic Bulletin \& Review, 4, 285-287.

Rosenthal, R., \& Rubin, D. (1985). Statistical analysis: Summarizing evidence versus establishing facts. Psychological Bulletin. 97. $527-529$

SERGENT, J. (1982). The cerebral balance of power: Confrontation or cooperation? Journal of Experimental Psychology: Human Perception \& Performance, 8, 253-272.

(Manuscript received October 23, 1996; accepted for publication November $1,1996$. 